\title{
Abnormal behavior in mice mutant for the Disc1 binding partner, Dixdc1
}

\author{
S Kivimäe ${ }^{1,5}$, P-M Martin ${ }^{1,5}$, D Kapfhamer ${ }^{2}$, Y Ruan ${ }^{1}$, U Heberlein ${ }^{2,3,4}$, JLR Rubenstein ${ }^{1,4}$ and BNR Cheyette ${ }^{1,4}$
}

Disrupted-in-Schizophrenia-1 (DISC1) is a genetic susceptibility locus for major mental illness, including schizophrenia and depression. The Disc1 protein was recently shown to interact with the Wnt signaling protein, DIX domain containing 1 (Dixdc1). Both proteins participate in neural progenitor proliferation dependent on Wnt signaling, and in neural migration independently of Wnt signaling. Interestingly, their effect on neural progenitor proliferation is additive. By analogy to Disc1, mutations in Dixdc1 may lead to abnormal behavior in mice, and to schizophrenia or depression in humans. To explore this hypothesis further, we generated mice mutant at the Dixdc1 locus and analyzed their behavior. Dixdc1 ${ }^{-1-}$ mice had normal prepulse inhibition, but displayed decreased spontaneous locomotor activity, abnormal behavior in the elevated plus maze and deficits in startle reactivity. Our results suggest that Dixdc1 $1^{-I-}$ mice will be a useful tool to elucidate molecular pathophysiology involving Disc1 in major mental illnesses.

Translational Psychiatry (2011) 1, e43; doi:10.1038/tp.2011.41; published online 27 September 2011

\section{Introduction}

Schizophrenia is a debilitating psychiatric disorder with a worldwide lifetime risk of approximately $1 \%$. It is thought to be caused by a combination of genetic factors and environmental insults. ${ }^{1,2}$ Several promising candidate susceptibility genes have been identified for schizophrenia, including NRG1, $D T N B P 1$ and DISC1. The dissection of molecular pathways centered on these candidate susceptibility genes is accordingly a major current priority for biological psychiatry that can lead to a better understanding of the pathogenesis in schizophrenia and possibly in other mental illnesses.

DISC1 was originally identified as a candidate susceptibility gene for major psychiatric disorders based on studies of a chromosomal translocation in a large Scottish family with a history of schizophrenia as well as major affective disorders. ${ }^{3}$ Genetic linkage studies have since implicated mutations in DISC1 as a risk factor for schizophrenia, schizoaffective disorder, bipolar disorder, major depression and the autism spectrum disorders. Several Disc1-mutant mouse lines have been engineered over the last 4 years. ${ }^{4-11}$ All these mouse lines display behavioral abnormalities, including in the cognitive and emotional realms, supporting a role for DISC1 mutations in the etiology of psychiatric disorders, and establishing Disc1 and the molecular pathways in which it participates as potential targets for diagnostic and therapeutic innovations.

Disc1 has multiple roles in neural development, including in morphogenesis, maturation, migration and synaptic integration of brain neurons $s^{12-14}$ through its interactions with several different protein partners. ${ }^{14-18}$ In a recently published study, Mao et al. ${ }^{19}$ discovered that among these partners is GSK3 $\beta$, a kinase central to the $\mathrm{Wnt} / \beta$-catenin signaling pathway that has independently been suggested to contribute to psychiatric pathophysiology. ${ }^{20,21}$ Disc1 enhances Wnt/ $\beta$-catenin-dependent neural progenitor proliferation by inhibiting GSK-3 $\beta$, thereby inducing $\beta$-catenin/TCF/LEF-dependent gene transcription. More recently, another Wnt signaling pathway regulator, Dixdc1 has been shown to form complexes with Disc1. ${ }^{22}$ Dixdc1, also called Ccd1, is a scaffold protein that possesses both Dishevelled-Axin (DIX) and coiled-coil protein-interaction domains that can act as a positive regulator of the Wnt/ $\beta$-catenin pathway. ${ }^{23}$ Concordant with the notion that Dixdc1, Disc1 and at least some forms of Wnt signaling cooperate in aspects of neural development relevant to psychiatric pathogenesis, knockdown of Dixdc1 in the mouse embryonic neocortex has been reported to result in a Wnt/ $\beta$ catenin-dependent reduction in neural progenitor cell proliferation, and a $W n t / \beta$-catenin-independent inhibition in neuronal migration. ${ }^{22}$

Given its molecular interactions with Disc1, and its role in both $\mathrm{Wnt} / \beta$-catenin-dependent and $\mathrm{Wnt} / \beta$-catenin-independent aspects of neural development, we hypothesized that DIXDC1 might contribute to pathophysiology underlying the abnormal behaviors in schizophrenia and other major psychiatric disorders. To explore this hypothesis further, we generated mice mutant at the Dixdc1 locus and analyzed their behavior.

\section{Materials and methods}

Generation of Dixdc1-mutant mice. To create the $5^{\prime}$ - and $3^{\prime}$-recombinogenic arms of a targeting construct, approximately

\footnotetext{
${ }^{1}$ Nina Ireland Laboratory of Developmental Neurobiology, Department of Psychiatry, University of California, San Francisco (UCSF), San Francisco, CA, USA; ${ }^{2}$ Ernest Gallo Clinic and Research Center, Emeryville, CA, USA; ${ }^{3}$ Department of Anatomy, UCSF, San Francisco, CA, USA and ${ }^{4}$ UCSF Graduate Program in Neuroscience, San Francisco, CA, USA

Correspondence: Dr BNR Cheyette, Department of Psychiatry, University of California, San Francisco (UCSF), San Francisco, CA 94158, USA.

E-mail: bc@ucsf.edu

${ }^{5}$ These authors contributed equally to this work.

Keywords: Disc1; locomotor activity; schizophrenia; startle reactivity; Wnt signaling

Received 20 June 2011; revised 13 July 2011; accepted 23 August 2011
} 
a
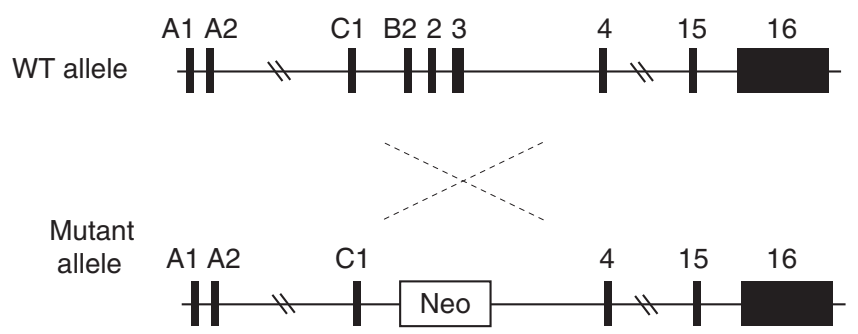

b

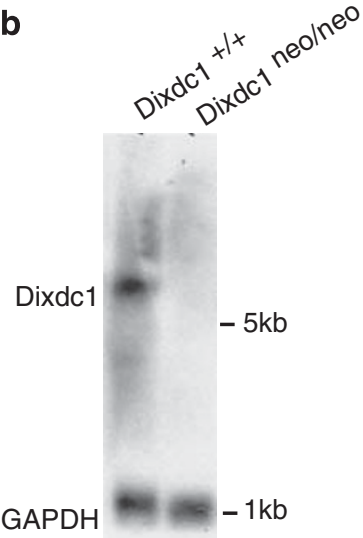

Figure 1 Generation of Dixdc1-mutant mice. (a) Schematic representation of Dixdc1 gene-targeting strategy. Exons B2, 2 and 3 were replaced by a neo cassette by homologous recombination, which is predicted to introduce a premature stop codon in all Dixdc1 transcripts. (b) Northern blot of postnatal cerebral cortical lysates using a probe complementary to most of the Dixdc1 coding sequence (Methods). GAPDH, glyceraldehyde-3-phosphate dehydrogenase; WT, wild type.

2.4 and $3.9 \mathrm{~kb}$, respectively, of the Dixdc1 locus from the $129 / \mathrm{Sv}$ mouse strain were inserted into pBluescript containing neomycin-positive selection and thymidine kinase-negative selection cassettes under PGK promoter control. Upon successful targeting, the neomycin expression cassette replaced exons $\mathrm{B} 2,2$ and 3 , and is therefore expected to disrupt all Dixdc1 splice forms ${ }^{24}$ (Figure 1). Correct targeting through homologous recombination in embryonic stem cells was confirmed by Southern blot and PCR. Mice carrying the targeted allele were generated using standard embryo manipulation and chimera breeding techniques. Genotyping of littermates from heterozygous intercrosses in the established colony was performed by genomic PCR using the following allele-specific primers: Neo-3.9: 5'-CATAGCGTTGGCTACCCGTGATATTG-3', and 3.9-Bam: 5'-GGAACTGTTGAGCTTTTTCCGGGAA-3', 3.9-4: 5'-GGTGATGGATTCGGAAAGAATTCCT-3'. PCR amplification was conducted for both alleles simultaneously, using all three primers in one reaction and the following parameters: 30 cycles of $94^{\circ} \mathrm{C}, 60 \mathrm{~s} ; 60^{\circ} \mathrm{C}, 120 \mathrm{~s}$ and $72^{\circ} \mathrm{C}, 60 \mathrm{~s}$. The wild type allele produces a 240-bp band, whereas the targeted allele produces a 340-bp band when resolved by electrophoresis in a standard agarose gel.

Northern blot analysis. A DNA probe corresponding to exons 3-16 of Dixdc1 was labeled with ${ }^{32} \mathrm{P}$-labeled dCTP through random priming, and then purified by precipitation. $20 \mu \mathrm{g}$ total RNA per lane, purified from cerebral cortex (postnatal day 6 ), was separated in $1 \%$ denaturing formaldehyde gel, blotted onto nylon membranes, and then hybridized. We used the following stringencies and times: hybridization overnight at $55^{\circ} \mathrm{C}$ in Ultrahyb buffer (Ambion, Austin, TX, USA), washing for $3 \times 15 \mathrm{~min}$ in $0.2 \times$ saline-sodium citrate buffer (SSC), $0.5 \%$ SDS (sodium dodecyl sulphate) at $68^{\circ} \mathrm{C}$. Exposure to film was overnight $(15 \mathrm{~h})$ at $-80^{\circ} \mathrm{C}$, with two intensification screens. Transcript sizing was performed by direct comparison with Millenium marker (Ambion, Austin, TX, USA) RNA ladder.

Behavioral tests. Male mutant mice $(n=10)$ and their wild type (WT) littermates $(n=9)$, born to heterozygous parents, underwent a series of behavioral tests (elevated plus maze, open field, forced swim, startle reactivity and prepulse inhibition (PPI), in that order, with a 7-day inter-test delay to allow the mice to rest) at the age of 3-4 months. For all tests, the experimenter had no knowledge of the genotype of the mice being tested and analyzed.

Elevated plus maze. The elevated plus maze is a commonly used test to assess anxiety-like behavior in rodents. ${ }^{25-27}$ The elevated plus maze apparatus consists of four alleys, two of which are open and the remaining two are enclosed, which intersect each other to form a cross or 'plus' (height: $41 \mathrm{~cm}$, arm length: $29 \mathrm{~cm}$, arm width: $9 \mathrm{~cm}$ ). Mice were placed in the center of the maze under dim ambient light conditions and allowed to explore freely for $5 \mathrm{~min}$. During this time, the mice were manually scored for the time they spent in the open arms (as defined by the placement of all four paws in one of the open arms).

Open field. Locomotor activity measurements were performed in Plexiglas locomotor activity chambers $(43 \times 43 \mathrm{~cm}$, Med Associates, St Albans, VT, USA), located in sound-attenuating cubicles equipped with a 2.8-W house light and exhaust fans that mask the external noise. The activity chambers contained two sets of 16 pulse-modulated infrared photobeams on opposing walls to record $x-y-z$ ambulatory movements, and were computer interfaced for data sampling at a $100-\mathrm{ms}$ temporal resolution. To measure the open-field activity, mice were placed individually in the center of a chamber and tracked for $60 \mathrm{~min}$. Data collected included horizontal activity (total horizontal distance, $\mathrm{cm}$ per $5 \mathrm{~min}$ ), vertical activity (counts of rearing), ambulatory episodes and average speed during ambulatory episodes ( $\mathrm{cm}$ second ${ }^{-1}$ ).

Forced swim test. The Porsolt or 'forced' swim test is an assay of 'behavioral despair' or depressive-like behavior in rodents that has been pharmacologically validated, particularly for selective serotonin reuptake inhibitors (SSRIs). ${ }^{28,29}$ Swim sessions were conducted by placing each subject mouse for $6 \mathrm{~min}$ in individual polycarbonate cylinders $(25.3 \mathrm{~cm}$ 
tall $\times 22.2 \mathrm{~cm}$ in diameter) filled with water $\left(23-25^{\circ} \mathrm{C}\right)$ to a depth of $15 \mathrm{~cm}$. Mice were scored manually for time immobile over this 6-min swim session. Immobility was defined as the absence of movement, except as necessary to keep afloat, including passive floating and turning in the absence of an active swimming motion. The water in the cylinder was changed for each subject. Immediately after testing, the animals were dried with a paper towel and placed in a recovery cage, half of which was positioned over an electric heat source, for a duration of $10 \mathrm{~min}$ before being returned to their homecage.

Acoustic startle reactivity (ASR)/ PPI. Both ASR and PPI were measured using the SR-Lab System (San Diego Instruments, San Diego, CA, USA). ${ }^{30}$ During all testing, the background was set at $70-\mathrm{dB}$ white noise. The ASR test consisted of eight trial types, each of which was presented five times in pseudorandom order. The trial types consisted of a single 40-ms startle stimulus of 90, 95, 100, 105, 110, 115, 120 or $125 \mathrm{~dB}$. Trials were presented with a variable intertrial interval of $15-20 \mathrm{~s}$. At the onset of each startle stimulus, maximum velocity $\left(V_{\max }\right)$ in arbitrary units was recorded every $1 \mathrm{~ms}$ for $65 \mathrm{~ms}$. The $V_{\max }$ was averaged across the trial types.

The PPI test consisted of a 5-min acclimation period, followed by seven trial types presented six times each in pseudorandom order. Each trial consisted of a 20-ms prepulse, an $80-\mathrm{ms}$ interval and a $40-\mathrm{ms}$ startle stimulus. Prepulse intensity was set at background, $74,78,82,86$ or $90 \mathrm{~dB}$, and the startle stimulus in each case was $120 \mathrm{~dB}$. One trial type consisted solely of background noise to control for general movement of the subject. Trials were presented with a variable intertrial interval of $15-20 \mathrm{~s}$. For each subject, the entire PPI test session lasted approximately $18 \mathrm{~min}$. At the onset of the startle stimulus, maximum velocity $\left(V_{\max }\right)$ in arbitrary units of the startle platform was recorded every $1 \mathrm{~ms}$ for $65 \mathrm{~ms}$. The $V_{\max }$ was averaged across trial type, with PPI calculated as the difference between $V_{\max }$ after prepulse trials $\left(V_{\max -\mathrm{PP}}\right)$ and $V_{\max }$ after startle stimulus alone trials $\left(V_{\max -\mathrm{ctl}}\right)$, and was expressed as a percentage of $V_{\text {max-ctl }}: \mathrm{PPI}=\left(\left(V_{\text {max-ctl }}\right)-\left(V_{\text {max-PP }}\right) /\left(V_{\max -\mathrm{ctl}}\right)\right)^{*} 100 \%$.

\section{Results and discussion}

Using homologous recombination technology, Dixdc1-mutant mice were genetically engineered in which exons B2, 2 and 3 were replaced by a neo cassette (Figure 1a). This mutation is predicted to introduce a premature stop codon altering all splice isoforms of Dixdc1. ${ }^{24}$ Dixdc1 $1^{\text {neo }}$ mice were backcrossed for three generations to the C57BL/6 isogenic mouse strain for use in this study; Dixdc $1^{\text {neo/neo }}$ mice are hereafter referred to as mutants (or $-/-$ in Figures). No Dixdc1 RNA transcript could be detected by Northern blot of brain lysates from mutant mice, validating Dixdc1 genetic disruption (Figure 1b). The mutants were born at Mendelian ratios, were viable and fertile, and were visually indistinguishable from their WT littermates. Gross morphology and size of the mutant neonatal brains were similar to WT, and analysis of neuronal subpopulations by in situ mRNA hybridization with Dlx1, Tbr1, Tle4, Pbx3 and Hef1 probes on cortical slices (embryonic day 16) did not reveal obvious differences between genotypes (data not shown).

In an open field, the mutant mice travelled significantly less distance (Figure 2a) and exhibited significantly less vertical activity counts than the WT (Figure $2 b$ ). Furthermore, the Dixdc $1^{-1-}$ mice were characterized by fewer ambulatory bouts than littermate controls (Figure 2c), whereas their average speed of locomotion during ambulatory bouts was normal (Figure 2d). These data suggest that the reduced activity of Dixdc1 $1^{-1-}$ mice is not due to a physical defect. Interestingly, decreased spontaneous locomotor activity in the open field contrasts with the hyperactivity observed in most Disc1 mutant models. ${ }^{9-11}$ In the forced swim test, mutants displayed a non-significant trend towards more time immobile than WT (Figure 3a) suggesting reduced motivation. In an elevated plus maze, Dixdc1 mutants spent significantly less time in the open arms than WT (Figure 3b),
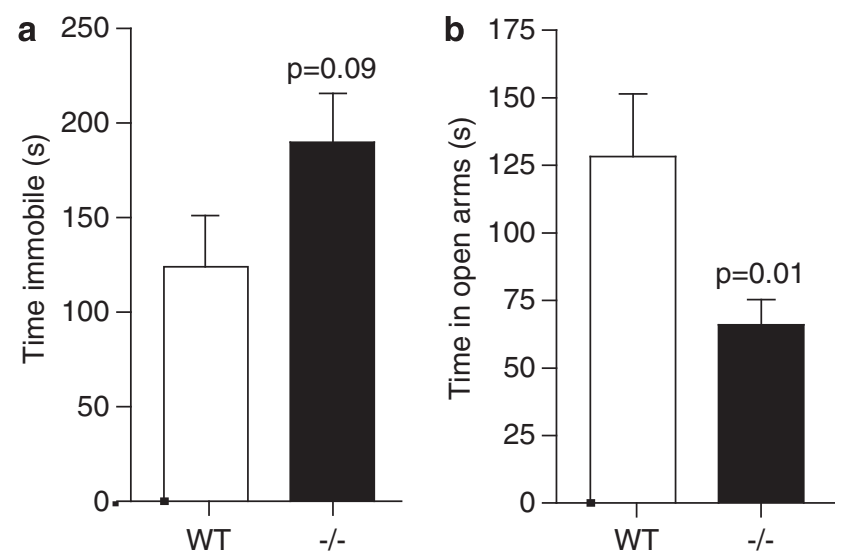


immobile in the forced swim test (Student's $t$-test). (b) Time in the open arms of the elevated plus-maze (Student's $t$-test). WT, wild type; p,probability value.
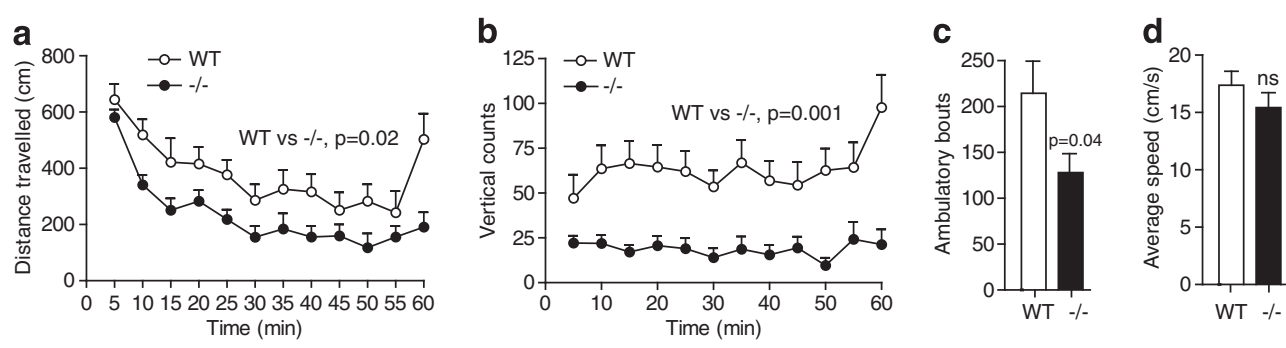

Figure 2 Spontaneous locomotion of Dixdc1 $1^{-1-}$ mice. (a) Locomotor activity in an open field (Repeated measures two-way analysis of variance (ANOVA), wild type (WT) vs $-/-, F(1,187)=5.88$ ). (b) Vertical counts in an open field (Repeated measures two-way ANOVA, WT vs $-/-, F(1,187)=13.76)$. (c) Number of ambulatory bouts (Student's $t$-test). (d) Average speed during ambulatory bouts (Student's $t$-test, $P=0.28)$. NS, not significant; $P$, probability value. 
a

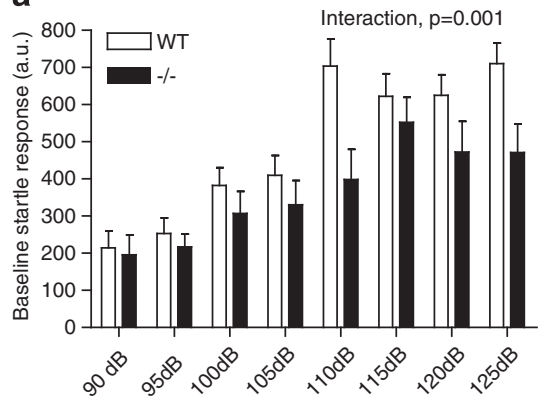

b

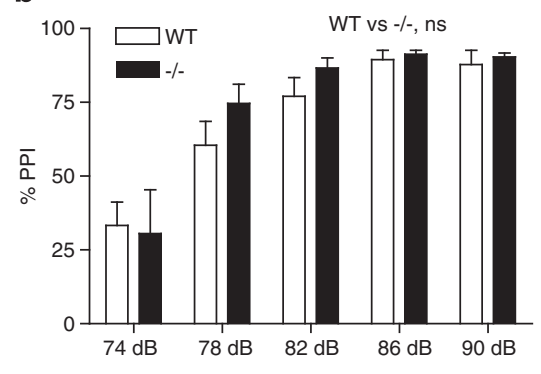

Figure 4 Baseline acoustic startle and prepulse inhibition (PPI) in Dixdc1 $1^{-1-}$ mice. (a) Acoustic startle response (Repeated measures two-way analysis of variance (ANOVA), Interaction, $F(7,119)=3.60$ followed by the Bonferroni multiple comparison test, $P<0.01$ for $110 \mathrm{~dB}$ ) (b) PPI of the acoustic startle response (Repeated measures two-way ANOVA, wild type (WT) vs mutant, $F(1,68)=0.47, P=0.47)$. NS, not significant.

consistent with increased anxiety. However, differences observed in both the forced swim test and the elevated plus maze may be confounded by the generally reduced locomotor activity documented in the open-field test. Resolution of this issue awaits more comprehensive behavioral phenotyping and analyses.

Similar to some Disc1 mutant models, ${ }^{11}$ the ASR in Dixdc1 mutants was abnormal; startle responses to tones $\geqslant 110 \mathrm{~dB}$ were decreased (significant difference at $110 \mathrm{~dB}$ only), whereas responses to tones $<110 \mathrm{~dB}$ were similar to the WT (Figure 4a). Nonetheless, in contrast to some Disc1mutant models and to other mouse models for schizophrenia, ${ }^{4,10,11,31}$ the PPI of the startle response was no different from WT in Dixdc1 mutants (Figure 4b).

To summarize, we have engineered a line of mice, which lacks Dixdc1, a partner of the Disc1 protein implicated in schizophrenia. Dixdc1-mutant mice are viable, fertile, have grossly normal brains at birth and display normal PPI, but have decreased spontaneous locomotor activity in an open field, abnormal behavior in the elevated plus maze and deficits in startle reactivity-a complex behavioral phenotype that though not immediately relatable to a specific psychiatric disorder nonetheless supports a role for this gene along with $D I S C 1$ in general psychiatric pathophysiology. One caveat to the present study is that the Dixdc $1^{\text {neo }}$ mice studied here were backcrossed only for three generations to the C57BL/6 isogenic mouse strain, whereas backcrosses for 10 generations or more are recommended to homogenize genetic background in comparative behavioral studies. Increasing the number of backcrosses would also reduce the chances of phenotypic effects arising from allelic differences at co-segregating flanking genes derived from the 129/Sv embryonic stem cell strain used for Dixdc1 gene targeting. In summary, further behavioral analyses in isogenic mice will be required to fully characterize the spectrum of sensory, affective and cognitive deficits in Dixdc1-mutants, but the present study highlights the potential utility of this novel mouse strain in elucidating roles of this gene, its partner Disc1 and related molecular pathways in the pathogenesis of behavioral disorders.

\section{Conflict of interest}

The authors declare no conflict of interest.
Acknowledgements. SK has received support from a NARSAD Young Investigator Award (Brain \& Behavior Research Foundation); this research has also been supported by a grant to BNRC from the Simons Foundation.

1. Chubb JE, Bradshaw NJ, Soares DC, Porteous DJ, Millar JK. The DISC locus in psychiatric illness. Mol Psychiatry 2008; 13: 36-64.

2. Jaaro-Peled H, Hayashi-Takagi A, Seshadri S, Kamiya A, Brandon NJ, Sawa A. Neurodevelopmental mechanisms of schizophrenia: understanding disturbed postnatal brain maturation through neuregulin-1-ErbB4 and DISC1. Trends Neurosci 2009; 32: 485-495.

3. Millar JK, Wilson-Annan JC, Anderson S, Christie S, Taylor MS, Semple CA et al. Disruption of two novel genes by a translocation co-segregating with schizophrenia. Hum Mol Genet 2000; 9: 1415-1423.

4. Niwa M, Kamiya A, Murai R, Kubo K, Gruber AJ, Tomita K et al. Knockdown of DISC1 by in utero gene transfer disturbs postnatal dopaminergic maturation in the frontal cortex and leads to adult behavioral deficits. Neuron 2010; 65: 480-489.

5. Ayhan Y, Abazyan B, Nomura J, Kim R, Ladenheim B, Krasnova IN et al. Differential effects of prenatal and postnatal expressions of mutant human DISC1 on neurobehavioral phenotypes in transgenic mice: evidence for neurodevelopmental origin of major psychiatric disorders. Mol Psychiatry 2011; 16: 293-306.

6. Shen S, Lang B, Nakamoto C, Zhang F, Pu J, Kuan SL et al. Schizophrenia-related neural and behavioral phenotypes in transgenic mice expressing truncated Disc1. J Neurosci 2008; 28: 10893-10904.

7. Kvajo M, McKellar H, Arguello PA, Drew LJ, Moore H, MacDermott AB et al. A mutation in mouse Disc1 that models a schizophrenia risk allele leads to specific alterations in neuronal architecture and cognition. Proc Natl Acad Sci USA 2008; 105: 7076-7081.

8. Li W, Zhou Y, Jentsch JD, Brown RA, Tian X, Ehninger D et al. Specific developmental disruption of disrupted-in-schizophrenia-1 function results in schizophrenia-related phenotypes in mice. Proc Natl Acad Sci USA 2007; 104: 18280-18285.

9. Pletnikov MV, Ayhan Y, Nikolskaia O, Xu Y, Ovanesov MV, Huang $\mathrm{H}$ et al. Inducible expression of mutant human DISC1 in mice is associated with brain and behavioral abnormalities reminiscent of schizophrenia. Mol Psychiatry 2008; 13: 173-186, 15.

10. Hikida T, Jaaro-Peled H, Seshadri S, Oishi K, Hookway C, Kong S et al. Dominant-negative DISC1 transgenic mice display schizophrenia-associated phenotypes detected by measures translatable to humans. Proc Natl Acad Sci USA 2007; 104: 14501-14506.

11. Clapcote SJ, Lipina TV, Millar JK, Mackie S, Christie S, Ogawa F et al. Behavioral phenotypes of Disc1 missense mutations in mice. Neuron 2007; 54: 387-402.

12. Duan X, Chang JH, Ge S, Faulkner RL, Kim JY, Kitabatake $Y$ et al. Disrupted-InSchizophrenia 1 regulates integration of newly generated neurons in the adult brain. Cell 2007; 130: 1146-1158.

13. Faulkner RL, Jang MH, Liu XB, Duan X, Sailor KA, Kim JY et al. Development of hippocampal mossy fiber synaptic outputs by new neurons in the adult brain. Proc Natl Acad Sci USA 2008; 105: 14157-14162.

14. Kamiya A, Kubo K, Tomoda T, Takaki M, Youn R, Ozeki Y et al. A schizophreniaassociated mutation of DISC1 perturbs cerebral cortex development. Nat Cell Biol 2005; 7: 1167-1178.

15. Enomoto A, Asai N, Namba T, Wang Y, Kato T, Tanaka M et al. Roles of disrupted-inschizophrenia 1-interacting protein girdin in postnatal development of the dentate gyrus. Neuron 2009; 63: 774-787.

16. Kim JY, Duan X, Liu CY, Jang MH, Guo JU, Pow-anpongkul N et al. DISC1 regulates new neuron development in the adult brain via modulation of AKT-mTOR signaling through KIAA1212. Neuron 2009; 63: 761-773.

17. Millar JK, Pickard BS, Mackie S, James R, Christie S, Buchanan SR et al. DISC1 and PDE4B are interacting genetic factors in schizophrenia that regulate cAMP signaling. Science 2005; 310: 1187-1191. 
18. Taya S, Shinoda T, Tsuboi D, Asaki J, Nagai $K$, Hikita T et al. DISC1 regulates the transport of the NUDEL/LIS1/14-3-3epsilon complex through kinesin-1. J Neurosci 2007; 27: 15-26.

19. Mao Y, Ge X, Frank CL, Madison JM, Koehler AN, Doud MK et al. Disrupted in schizophrenia 1 regulates neuronal progenitor proliferation via modulation of GSK3beta/ beta-catenin signaling. Cell 2009; 136: 1017-1031.

20. De Ferrari GV, Moon RT. The ups and downs of Wnt signaling in prevalent neurological disorders. Oncogene 2006; 25: 7545-7553.

21. Okerlund ND, Cheyette BN. Synaptic Wnt signaling-a contributor to major psychiatric disorders? J Neurodev Disord 2011; 3: 162-174.

22. Singh KK, Ge X, Mao Y, Drane L, Meletis K, Samuels BA et al. Dixdc1 is a critical regulator of DISC1 and embryonic cortical development. Neuron 2010; 67: 33-48.

23. Shiomi K, Uchida H, Keino-Masu K, Masu M. Ccd1, a novel protein with a DIX domain, is a positive regulator in the Wnt signaling during zebrafish neural patterning. Curr Biol 2003; 13: 73-77.

24. Shiomi K, Kanemoto M, Keino-Masu K, Yoshida S, Soma K, Masu M. Identification and differential expression of multiple isoforms of mouse Coiled-coil-DIX1 (Ccd1), a positive regulator of Wnt signaling. Brain Res Mol Brain Res 2005; 135: 169-180.

25. Lister RG. The use of a plus-maze to measure anxiety in the mouse. Psychopharmacology (Berl) 1987; 92: 180-185.

26. Belzung $C$, Griebel $G$. Measuring normal and pathological anxiety-like behaviour in mice: a review. Behav Brain Res 2001; 125: 141-149.
27. File SE. Factors controlling measures of anxiety and responses to novelty in the mouse. Behav Brain Res 2001; 125: 151-157.

28. Crowley JJ, Jones MD, O'Leary OF, Lucki I. Automated tests for measuring the effects of antidepressants in mice. Pharmacol Biochem Behav 2004; 78: 269-274.

29. Cryan JF, Valentino RJ, Lucki I. Assessing substrates underlying the behavioral effects of antidepressants using the modified rat forced swimming test. Neurosci Biobehav Rev 2005; 29: 547-569.

30. Tarantino LM, Gould TJ, Druhan JP, Bucan M. Behavior and mutagenesis screens: the importance of baseline analysis of inbred strains. Mamm Genome 2000; 11: 555-564.

31. Wen L, Lu YS, Zhu XH, Li XM, Woo RS, Chen YJ et al. Neuregulin 1 regulates pyramidal neuron activity via ErbB4 in parvalbumin-positive interneurons. Proc Natl Acad Sci USA 2010; 107: 1211-1216.

Translational Psychiatry is an open-access journal published by Nature Publishing Group. This work is licensed under the Creative Commons Attribution-Noncommercial-No Derivative Works 3.0 Unported License. To view a copy of this license, visit http://creativecommons.org/licenses/by-nc-nd/3.0/ 